Copyright by the American Institute of Physics (AIP). Dickerson, BD; Irving, DM; Herz, E; et al., "Synthesis kinetics of CdSe quantum dots in trioctylphosphine oxide and in stearic acid," Appl. Phys. Lett. 86, 171915 (2005); http:// dx.doi.org/10.1063/1.1921347

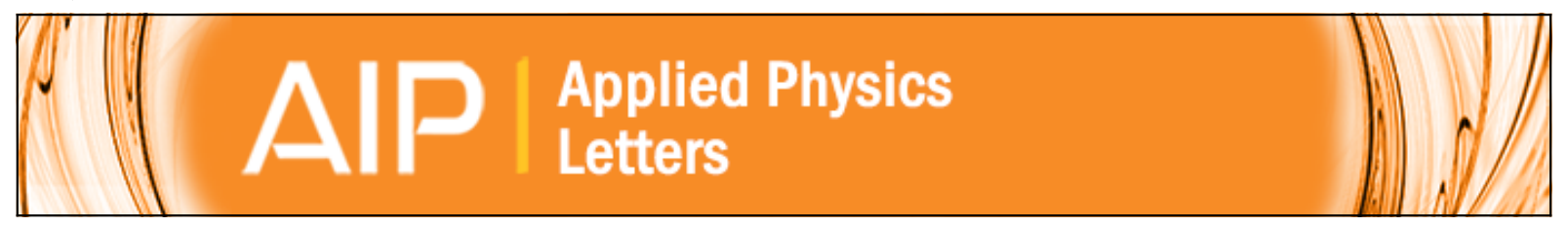

Synthesis kinetics of CdSe quantum dots in trioctylphosphine oxide and in stearic acid

B. D. Dickerson, D. M. Irving, E. Herz, R. O. Claus, W. B. Spillman Jr., and K. E. Meissner

Citation: Applied Physics Letters 86, 171915 (2005); doi: 10.1063/1.1921347

View online: http://dx.doi.org/10.1063/1.1921347

View Table of Contents: http://scitation.aip.org/content/aip/journal/apl/86/17?ver=pdfcov

Published by the AIP Publishing

Over 700 papers \&

presentations on

multiphysics simulation new now

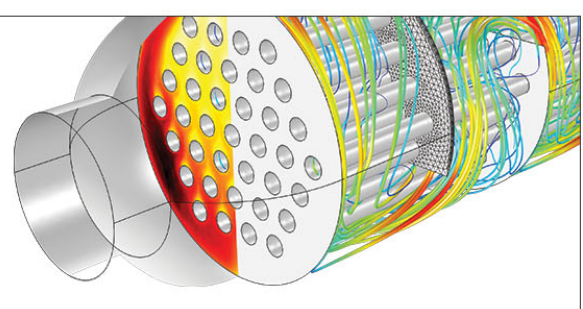




\title{
Synthesis kinetics of CdSe quantum dots in trioctylphosphine oxide and in stearic acid
}

\author{
B. D. Dickerson, ${ }^{\text {a) }}$ D. M. Irving, E. Herz, R. O. Claus, and W. B. Spillman, Jr. \\ Virginia Tech Applied Biosciences Center, Virginia Polytechnic Institute and State University, Blacksburg, \\ Virginia 24061-0356 \\ K. E. Meissner \\ Department of Biomedical Engineering, Texas A\&M University, College Station, Texas 77843
}

(Received 12 January 2005; accepted 15 March 2005; published online 22 April 2005)

\begin{abstract}
A diffusion-barrier model described the early evolution of size-dependent photoluminescence emission from CdSe quantum dots formed by organometallic synthesis. Emission peak widths, emission redshift rates, and nanocrystal growth rates all decreased to a minimum at a reaction completion time. Growth after the completion time by Ostwald ripening was marked by a doubling of the activation energy. The temperature dependence of both reaction completion rates and photoluminescence redshift rates followed Arrhenius behavior governed by activation energies that increased with solvent molecular weight, in this limited case. In stearic acid and in trioctylphosphine oxide, the typical activation energies were $0.6 \pm 0.1$ and $0.92 \pm 0.26 \mathrm{eV} /$ molecule, respectively.
\end{abstract}

(C) 2005 American Institute of Physics. [DOI: 10.1063/1.1921347]

Photoluminescence (PL) emission from colloidal CdSe quantum dots (QDs) can be adjusted within the visible spectrum from 475 to $645 \mathrm{~nm}$. This versatility leads to numerous photonic applications, such as optical fiber amplifiers, ${ }^{1,2}$ color displays using light-emitting diode arrays, ${ }^{3}$ optical temperature probes, ${ }^{4}$ and biomedical imaging. ${ }^{5}$ Conjugation of QDs with antibodies yields biomarkers that compete with traditional organic fluorescent tags in terms of biocompatibility, excitation and filtering simplicity, and photostability. ${ }^{6,7}$ All of these applications prefer sharp emission peaks and high photoluminescence quantum yield. ${ }^{8}$

Since QD emission is size dependent, colloidal growth must be carefully controlled to achieve the target average radius with a narrow particle size distribution. Organometallic synthesis routes were pioneered ${ }^{9}$ and modified ${ }^{10}$ to produce superior crystallinity and higher photoluminescence quantum yield $^{8,11}$ from hotter reactions, and to provide better surface passivation, ${ }^{11,12}$ all by growing QDs in trioctylphosphine oxide (TOPO) rather than in water. Peng \& Peng observed that the reaction solvent strongly affected synthesis kinetics. ${ }^{13}$

Early theoretical studies of nanocrystal growth rates emphasized Ostwald ripening, ${ }^{14-17}$ whereby larger particles grow and smaller particles dissolve, thus widening particle size distributions when reactants are scarce. Researchers concluded that narrower size distributions could be achieved when nanocrystal growth was limited by diffusion rather than by reaction rate. ${ }^{18,19}$ Intuitively, the Gibbs-Thompson equation helped explain why size distributions could widen with synthesis time, if reactant concentrations were depleted below the QDs' size-dependent solubility threshold. ${ }^{19-21}$ From this, Weller's group developed a comprehensive model with several activation energies to simulate QD size evolution under a wide variety of organometallic synthesis conditions. ${ }^{18}$ In the limiting case of diffusion-controlled growth before Ostwald ripening, organometallic synthesis kinetics should be characterized by a single activation energy

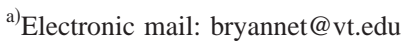

$Q$. Traditionally, an activation energy would be extracted from the slope in an Arrhenius plot of $\ln ($ rate $)$ versus $1 / T$, where $T$ is the absolute temperature. Recently, a study of CdSe QDs grown in stearic acid (SA) used Arrhenius analysis to estimate $Q$ from observed PL redshift rates (i.e., the changes in PL peak wavelength per change in reaction time between sequential samples). ${ }^{22}$

In this letter, redshift rates and reaction completion rates are used to compare activation energies for CdSe QD synthesis in TOPO versus SA. Although the values of $Q$ are specific for each materials system, the methods of estimating $Q$ and interpreting PL data to track QD growth are general enough in principle to study the synthesis kinetics of many type I binary semiconductor QDs in various organic solvents.

CdSe nanocrystals grew faster in SA than in TOPO, as shown by higher redshift rates in Fig. 1. In this Arrhenius plot, the activation energy was proportional to the slope of a linear fit through data from each solvent. Using redshift rates, estimates of $Q$ varied with test wavelength, as shown

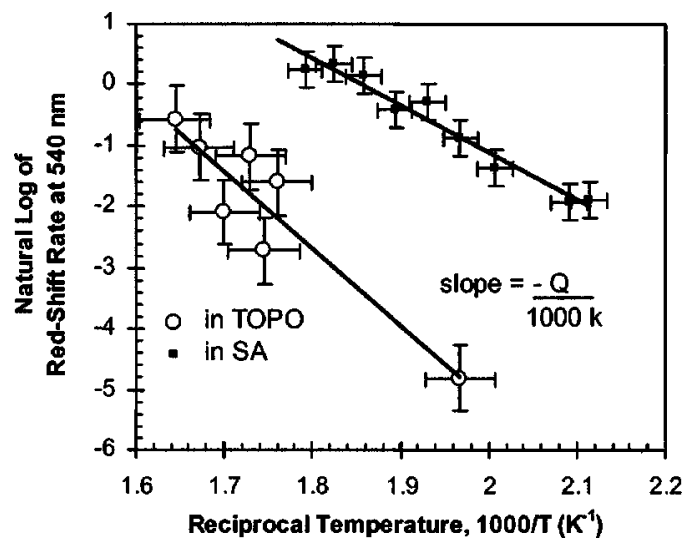

FIG. 1. Arrhenius plot showing the temperature dependence of PL redshift rates observed at a test wavelength of $540 \mathrm{~nm}$ for CdSe QDs grown in stearic acid (SA) and trioctylphosphine oxide (TOPO). The activation energy for diffusion in each solvent is proportional to the slope of the best line through each data set. 


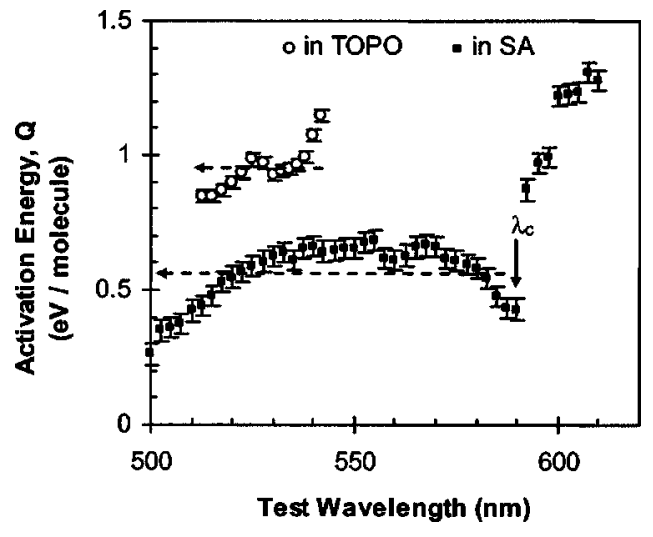

FIG. 2. Activation energy estimates from redshift rates at different test wavelengths for CdSe QD growth in TOPO and in SA. The average activation energies are as follows: $Q_{\mathrm{TOPO}}=0.95 \pm 0.27 \mathrm{eV} / \mathrm{molecule}$ and $Q_{\mathrm{SA}}$ $=0.56 \pm 0.12 \mathrm{eV} /$ molecule. In SA, the doubling of $Q$ above $590 \mathrm{~nm}$ is consistent with the onset of Ostwald ripening at $\lambda_{c}$.

in Fig. 2, but the average activation energies $\left(Q_{\mathrm{TOPO}}\right.$ $=0.95 \pm 0.27 \mathrm{eV} /$ molecule versus $Q_{\mathrm{SA}}=0.56 \pm 0.12 \mathrm{eV} /$ molecule) increased with the solvent's molecular weight $\left(W_{\mathrm{TOPO}}=386.65 \mathrm{~g} / \mathrm{mole}\right.$ versus $\left.W_{\mathrm{SA}}=284.47 \mathrm{~g} / \mathrm{mole}\right)$. A similar trend of faster QD growth in smaller molecular weight solvents was observed within the fatty acid solvent system. ${ }^{23}$ In our case, the chelating functional groups of TOPO and SA were different, so separate metal-organic interaction energies might also have contributed to each activation energy.

The synthesis of CdSe QDs in TOPO followed conventional methods with $\mathrm{CdO}$ as a precursor. ${ }^{10}$ For every mole of $\mathrm{CdO}$, there were 24.42 moles of TOPO with 0.509 moles of tetradecylphosphonic acid, and 1.30 moles of Se dissolved in 13.46 moles of trioctylphosphine. For samples referred to as grown "in SA," the $3.777 \mathrm{~g}$ of TOPO in a standard batch was replaced with an equal mass of a mixture of $95 \mathrm{wt} . \%$ SA and 5 wt. $\%$ TOPO. The $5 \mathrm{wt} . \%$ of TOPO was included to insure there would be enough coordinating ligand, just in case stearic acid might not perform this function. Several reaction temperatures between 205 and $335{ }^{\circ} \mathrm{C}$ were used. Samples of the hot reaction liquid were extracted at progressively doubling time intervals, quenched in methanol, then resuspended and diluted in toluene. Using a Hitachi F-4500 fluorescence spectrophotometer, PL spectra were acquired at room temperature with excitation at $370 \mathrm{~nm}$.

A diffusion-limited model provides a framework for analysis of PL data. The model only simulates QD growth after nucleation and before Ostwald ripening, and it assumes that the number of nuclei remain constant during this period. ${ }^{24}$ In that case, the average growth rate $v_{(t)}$ (in moles $\mathrm{s}^{-1}$ ), of each QD is approximated by Eq. (1), where $Q$ is the activation energy (in $\mathrm{eV}$ molecule ${ }^{-1}$ ), $T$ is temperature (in $\mathrm{K}$ ), and $k$ is Boltzmann's constant (in $\mathrm{eV}$ molecule ${ }^{-1} \mathrm{~K}^{-1}$ ), $R_{(t)}$ is the average QD radius (in $\mathrm{cm}$ ), $\left[M_{(t)}\right]$ is the molar concentration (in moles $\mathrm{cm}^{-3}$ ) of the limiting reactant (in this case organometallic Cd complexes), $D_{o}$ is the diffusion coefficient (in $\mathrm{cm}^{2} \mathrm{~s}^{-1}$ ), and $L_{D}$ is a characteristic diffusion length (in $\mathrm{cm}$ ), which might represent the ligand barrier thickness or a reactant depletion distance in the reaction solvent:

$$
v_{(t)}=4 \pi R_{(t)}^{2} \frac{\left[M_{(t)}\right]}{L_{D}} D_{\partial} \exp \left\{-\frac{Q}{k T}\right\}
$$

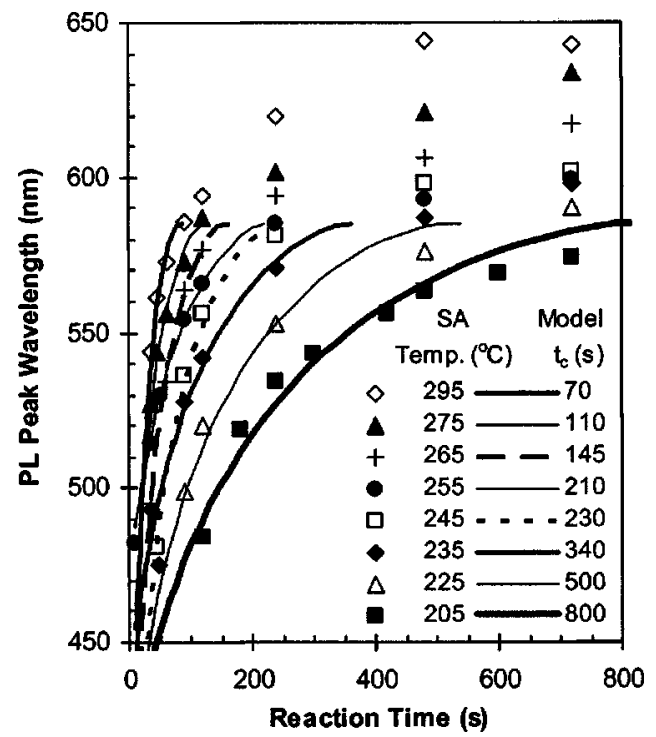

FIG. 3. Evolution of PL peak emission wavelength with reaction time for CdSe QDs synthesized in stearic acid. Curves show the modeled PL evolution, with the best fit completion time $t_{c}$, indicated for each reaction temperature.

Theoretically, Arrhenius analysis of redshifts is based on the first-order approximation that $v_{(t)}$ is proportional to $d \lambda / d t$, where $\lambda$ is the PL emission peak wavelength (in $\mathrm{nm}$ ) and $t$ is the reaction time (in s). This result (2) can be derived from the effective mass approximation for spherical quantum $\operatorname{dots}^{25}$ after differentiation:

$$
d \lambda / d t \propto v_{(t)} \lambda^{2} / R^{5} .
$$

To the degree that Eq. (2) is accurate, it is convenient to monitor the redshift rate as a way to track QD growth rate. To estimate $Q$ from $d \lambda / d t$, redshift rates at different temperatures are compared at a common test wavelength. Equation (2) shows how fixing $\lambda$ minimizes sensitivity to $R$. However, the growth rate also depends on $\left[M_{(t)}\right]$, so that systematic variation of $Q$ with test wavelength, seen in Fig. 2, may be due to different rates of reactant depletion at different temperatures.

Experimentally, redshift rates and PL peak widths both tend to decrease to a minimum as $\lambda$ approaches a completion wavelength $\lambda_{c}$, independent of synthesis temperature. For these QDs grown in SA, $\lambda_{c}$ is $590 \pm 5 \mathrm{~nm}$. Coincidentally, in Fig. 2, as the test wavelength is moved above $590 \mathrm{~nm}$, the estimate of $Q_{\mathrm{SA}}$ for growth in SA doubles sharply to $1.25 \pm 0.05 \mathrm{eV} /$ molecule, suggesting a change in the growth mechanism. All of these observations are consistent with the exhaustion of reactants at $t_{c}$, followed by the onset of Ostwald ripening. For $\mathrm{CdSe}$ QDs grown in TOPO, $\lambda_{c}$ is $560 \pm 5 \mathrm{~nm}$. However $Q_{\text {TOPO }}$ could not be accurately estimated from test wavelengths near $560 \mathrm{~nm}$ due to a limited range of chosen synthesis temperatures.

To avoid systematic variations in $Q$ with test wavelength, an alternative analysis method is developed. When $v_{(t)},\left[M_{(t)}\right]$, and $R_{(t)}$ are all expressed in terms of $f_{(t)}$ (the remaining fraction of the original moles of limited reactant), then Eq. (1) becomes a differential equation (3), where $A_{(T)}$ is independent of $t$ and $f$. Integrating Eq. (3) from an intermediate time after nucleation $t_{i}$ to a given reaction time $t$ yields an expression (4) describing how reactants are gradu- 


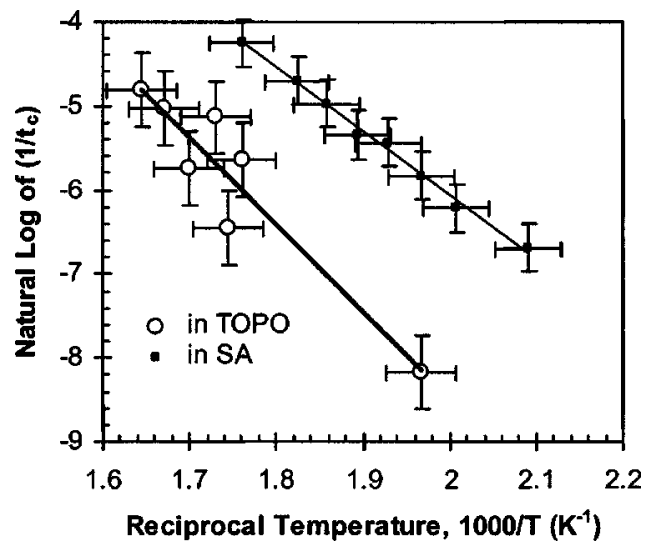

FIG. 4. Temperature dependence of reaction completion rate $1 / t_{c}$. From the slopes, $Q_{\mathrm{SA}}$ is $0.65 \pm 0.07 \mathrm{eV} /$ molecule and $Q_{\mathrm{TOPO}}$ is $0.89 \pm 0.25 \mathrm{eV} /$ molecule for CdSe quantum dots grown in stearic acid and in trioctylphosphine oxide, respectively.

ally depleted over a reaction completion time $t_{c}$. The intermediate precursor fraction $f_{i}$ is less than 1.0 because some reactants are consumed during nucleation: ${ }^{21}$

$$
\begin{aligned}
& d f / d t=-A_{(T)} f^{1 / 3}, \\
& f_{(t)}=f_{i}\left(1-\left\{t-t_{i}\right\} / t_{c}\right)^{3 / 2} .
\end{aligned}
$$

Growth of the average QD radius (5) can be derived geometrically from Eq. (4), assuming reactants are consumed only to produce QDs. Here, $N$ is the number of nuclei, $M_{o}$ is the original number of moles of limited reactant, $W_{\mathrm{CdSe}}$ is the molecular weight of CdSe (in $\mathrm{g} / \mathrm{mole}$ ), and $\rho$ is the density of CdSe (in $\mathrm{g} \mathrm{cm}^{-3}$ ):

$$
R_{(t)}=\left(\frac{\left(1-f_{(t)}\right) M_{o} 3 W_{\mathrm{CdSe}}}{4 \pi N \rho}\right)^{1 / 3} .
$$

To convert average QD radius to modeled PL photon wavelength (6), the effective mass approximation ${ }^{23}$ is used, despite its limitations. ${ }^{9}$ In this case, it is not necessary to know effective carrier masses or $N$, since they are contained within one parameter, the completion wavelength $\lambda_{c}$, which is readily determined by analyzing PL data. In Eq. (6), $h c$ is Planck's constant times the speed of light:

$$
\lambda_{(t)}=\frac{h c}{E_{g}+\left(h c / \lambda_{c}-E_{g}\right)\left(1-f_{(t)}\right)^{-2 / 3}} .
$$

Activation energies were also estimated from the temperature dependence of $t_{c}$. Experimental observations of $\lambda$ versus $t$ were compared to modeled curves (6) to estimate $t_{c}$ at each temperature (Fig. 3). $Q$ was then calculated from the slope in a plot of $\ln \left(1 / t_{c}\right)$ versus $1000 / T$, shown in Fig. 4. The activation energies calculated using $t_{c}\left(Q_{\mathrm{SA}}\right.$ $=0.65 \pm 0.07 \mathrm{eV} /$ molecule and $Q_{\mathrm{TOPO}}=0.89 \pm 0.25 \mathrm{eV} /$ molecule) agree with those estimated using redshift rates, within experimental error. Estimating activation energies from the temperature dependence of completion rates, rather than from redshift rates, reduces random and systematic errors by analyzing overall trends rather than local trends in PL evolution.

To summarize, growth of CdSe quantum dots during the early stages of organometallic synthesis was described by an analytical model of the diffusion and depletion of reactants. PL spectra were a convenient way to track the Arrhenius behavior of QD growth rates. Comparing growth in different coordinating solvents revealed that the diffusion activation energy increased with the solvent's molecular weight, in the case of SA versus TOPO. Compared to growth in TOPO, CdSe nanocrystals grew more rapidly in SA and produced narrow PL emission peaks at longer wavelengths, before the onset of Ostwald ripening.

The authors would like to thank Dr. You-Xiong Wang and Dr. Richey Davis for their helpful suggestions regarding colloidal chemistry. Financial support for this project was provided by the Virginia Tech Applied Biosciences Center.

${ }^{1}$ S. V. Kershaw, M. T. Harrison, and M. G. Burt, Philos. Trans. R. Soc. London, Ser. A 361, 331 (2003).

${ }^{2}$ K. E. Meissner and C. Holton, in Proceedings of Quantum Dots 2004 (Institute for Microstructural Sciences, National Research Council of Canada, Ottawa, 2004), p. 111.

${ }^{3}$ J. Lee, V. C. Sundar, J. R. Heine, M. G. Bawendi, and K. F. Jense, Adv. Mater. (Weinheim, Ger.) 12, 1102 (2000).

${ }^{4}$ G. W. Walker, V. C. Sundar, C. M. Rudzinski, A. W. Wun, M. G. Bawendi, and D. G. Nocera, Appl. Phys. Lett. 83, 3555 (2003).

${ }^{5}$ X. Gao, Y. Cui, R. M. Levenson, L. W. K. Chung, and S. Nie, Nat. Biotechnol. 22, 969 (2004).

${ }^{6}$ B. D. Butkus, Biophotonics Int. 11, 34 (2004).

${ }^{7}$ A. M. Derfus, C. W. W. Chan, and N. Bhatia, Nano Lett. 4, 11 (2004).

${ }^{8}$ N. Gaponik, D. V. Talapin, A. L. Rogach, K. Hoppe, E. V. Shevchenko, A. Kornowski, A. Eychmuller, and H. Weller, J. Phys. Chem. B 106, 7177 (2002).

${ }^{9}$ C. B. Murray, D. J. Norris, and M. G. Bawendi, J. Am. Chem. Soc. 115, 8706 (1993).

${ }^{10}$ Z. A. Peng and X. Peng, J. Am. Chem. Soc. 123, 183 (2001).

${ }^{11}$ D. V. Talapin, S. Haubold, A. L. Rogach, A. Kornowski, M. Haase, and H. Weller, J. Phys. Chem. B 105, 2260 (2001).

${ }^{12}$ D. V. Talapin, Andrey L. Rogach, I. Mekis, S. Haubold, A. Kornowaski, M. Haase, and H. Weller, Colloids Surf., A 202, 145 (2002).

${ }^{13}$ Z. A. Peng and X. Peng, J. Am. Chem. Soc. 123, 1389 (2001).

${ }^{14}$ J. A. Marqusee and J. Ross, J. Chem. Phys. 80, 535 (1984).

${ }^{15}$ G. Venzl, Phys. Rev. A 31, 3431 (1985).

${ }^{16}$ J. H. Yao, K. R. Elder, H. Guo, and M. Grant, Phys. Rev. B 47, 14110 (1993).

${ }^{17}$ S. Egelhaaf, U. Olsson, P. Schurtenberger, J. Morris, and H. Wennerstrom, Phys. Rev. E 60, 5681 (1999).

${ }^{18}$ D. V. Talapin, A. L. Rogach, M. Haase, and H. Weller, J. Phys. Chem. B 105, 12278 (2001).

${ }^{19}$ X. Peng, J. Wickman, and A. P. Alivisatos, J. Am. Chem. Soc. 120, 5343 (1998).

${ }^{20}$ T. Sugimoto, Adv. Colloid Interface Sci. 28, 65 (1987).

${ }^{21}$ Z. A. Peng and X. G. Peng, J. Am. Chem. Soc. 124, 3343 (2002).

${ }^{22}$ B. D. Dickerson, D. M. Irving, E. Herz, K. E. Meissner, R. O. Claus, and W. B. Spillman, Jr., in Proceedings of Optics in the Southeast, OISE 2004 (SPIE, Charlotte, NC, 2004), p. 69.

${ }^{23}$ L. Qu, Z. A. Peng, and X. Peng, Nano Lett. 1, 333 (2001).

${ }^{24}$ C. R. Bullen and P. Mulvaney, Nano Lett. 4, 2303 (2004).

${ }^{25} \mathrm{H}$. Haug and S. W. Koch, Quantum Theory of the Optical and Electronic Properties of Semiconductors, 3rd ed. (World Scientific, River Edge, NJ, 1990), pp. 386-399. 